This item was submitted to Loughborough's Research Repository by the author.

Items in Figshare are protected by copyright, with all rights reserved, unless otherwise indicated.

\title{
A method for obtaining three-dimensional data on ski jumping using pan and tilt cameras
}

\section{PLEASE CITE THE PUBLISHED VERSION}

\section{PUBLISHER}

(c) Human Kinetics

\section{VERSION}

VoR (Version of Record)

\section{LICENCE}

CC BY-NC-ND 4.0

\section{REPOSITORY RECORD}

Yeadon, Maurice R.. 2019. "A Method for Obtaining Three-dimensional Data on Ski Jumping Using Pan and Tilt Cameras". figshare. https://hdl.handle.net/2134/8684. 
This item was submitted to Loughborough's Institutional Repository (https://dspace.lboro.ac.uk/) by the author and is made available under the following Creative Commons Licence conditions.

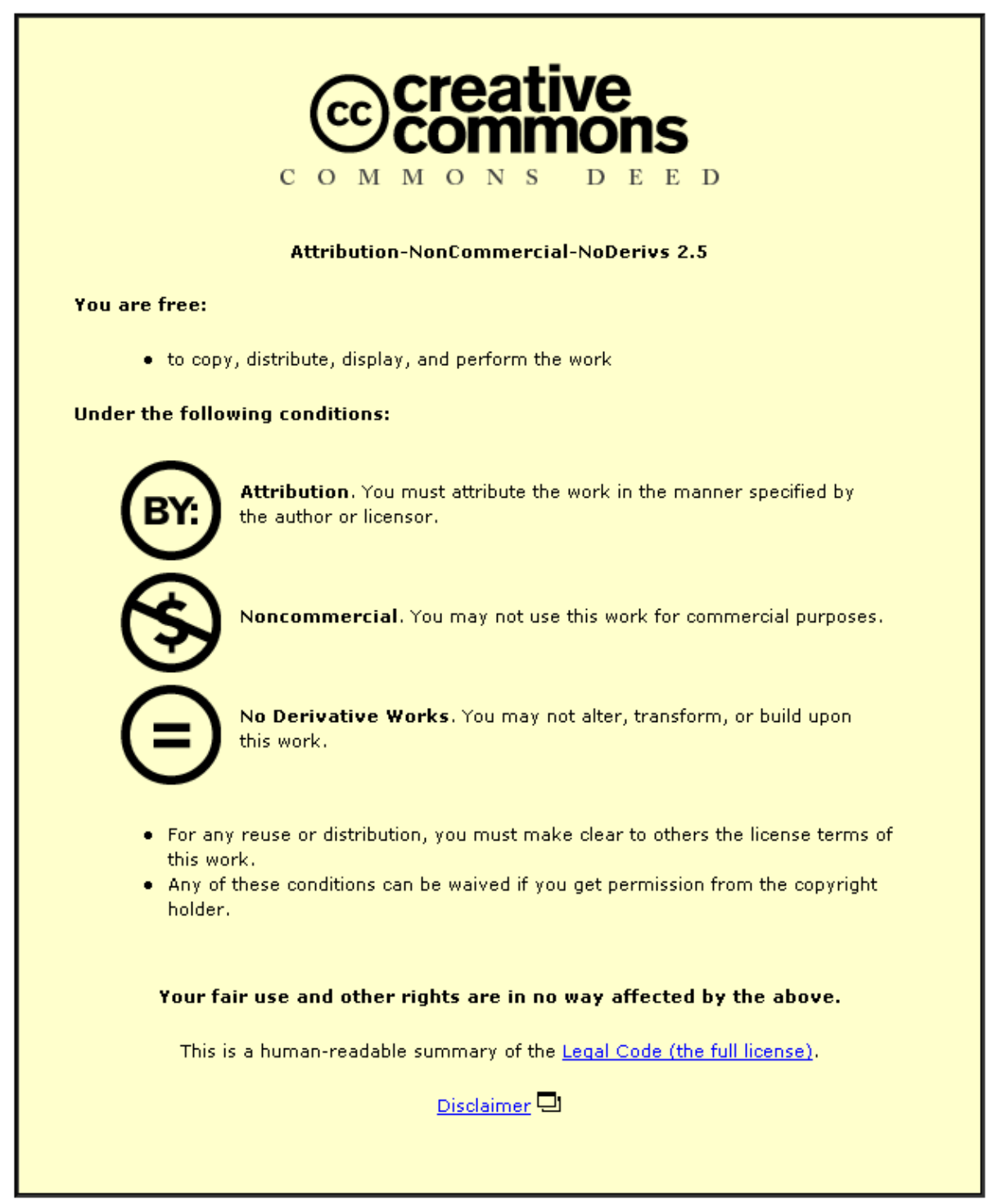

For the full text of this licence, please go to: http://creativecommons.org/licenses/by-nc-nd/2.5/ 


\title{
A Method for Obtaining Three-Dimensional Data on Ski Jumping Using Pan and Tilt Cameras
}

\author{
Maurice R. Yeadon
}

\begin{abstract}
A method is presented for the three-dimensional analysis of ski jumping using two pan and tilt cameras. In each film frame two reference markers are digitized and identified so that a pseudo focal length and three angles defining camera orientation can be calculated from a knowledge of the positions of camera and markers. In each film frame 12 body landmarks are digitized and the films taken by the two cameras are synchronized using the digitized displacement data. The time histories of the center of mass location and 15 angles describing the orientation and configuration of the jumper are calculated. Digitization errors lead to an error of $0.05 \mathrm{~m}$ in center of mass location and an error of $1^{\circ}$ in orientation angles.
\end{abstract}

Ski jumping may be thought of as an activity in which the body moves in a vertical plane and maintains configurational symmetry about that plane. This concept suggests that a three-dimensional analysis will yield no more relevant information than a two-dimensional analysis. Even for a jump in which perfect symmetry is maintained, this is not the case. The mediolateral separation of the skis and their orientation about the longitudinal axes of the legs affect the frontal area presented and may be an important element of technique. In fact many ski jumpers do not maintain such symmetry but turn their skis to one side during flight until just before landing, at which point the skis are realigned. In order to investigate such asymmetries of technique, a method of three-dimensional film analysis is required.

There are two types of methods for three-dimensional cinematography that use two or more cameras having fixed locations and fixed orientations. In the first type, the locations of the cameras and reference markers are determined using surveying techniques prior to filming (Bergemann, 1974; Penrose, Wood, \& Blanksby, 1976). In the seond type, the reference markers take the form of a known control object and the camera locations are inferred from the images of the control object (Abdel-Aziz \& Karara, 1971; Van Gheluwe, 1978). Such

M.R. Yeadon is with the Biomechanics Laboratory at the University of Calgary, 2500 University Drive N.W., Calgary, Alberta, Canada T2N 1 N4. 
methods using fixed cameras are suitable for obtaining three-dimensional information on human movement if the field of view is not more than 10 meters. As the field of view increases beyond this value, the image size of the athlete decreases so that it becomes more difficult to identify body landmarks with accuracy.

In sports for which the primary movement is horizontal, the problem of image size may be solved by using horizontally panning cameras. Dapena (1978) developed a method of three-dimensional cinematography using two cameras that were free to rotate about vertical axes. In ski jumping, the vertical displacements are in excess of 30 meters, so it is necessary to use tripods with pan and tilt heads that permit cameras to rotate about both vertical and horizontal axes.

In order to determine the orientations of such cameras it is necessary to have reference markers visible in each film frame. Since each reference marker that is digitized must also be identified by number, it is desirable to minimize the number of markers required for each frame while still obtaining accurate camera orientations. This paper presents a method of three-dimensional cinematography of ski jumping using pan and tilt cameras whereby two reference markers are used in each film frame.

\section{Theory}

\section{Methods}

The orientation of a right triad $c_{1}, c_{2}, c_{3}$ of unit vectors, defining the frame of reference of camera $\mathrm{C}$, may be defined relative to an inertial frame I comprising the triad $i_{1}, i_{2}, i_{3}$ in the following way: If frame $\mathrm{C}$ is initially aligned with frame $\mathrm{I}$, then successive rotations through angles $\psi_{\mathrm{c}}, \theta_{\mathrm{c}}, \phi_{\mathrm{c}}$ about axes $c_{3}, c_{2}, c_{1}$ bring frame $\mathrm{C}$ into its final orientation (Figure 1). These angles will permit transformations from the inertial frame I and the camera or digitizer frame $\mathrm{C}$. If the tripod pan and tilt head is initially leveled to be exactly horizontal, and the projection head of the digitizer is aligned so that the film frame edges are exactly parallel to the digitizer axes, the screw angle $\theta_{\mathrm{c}}$ will be zero. In this case the orientation of frame $\mathrm{C}$ will be completely specified by the pan angle $\psi_{\mathrm{c}}$ and the tilt angle

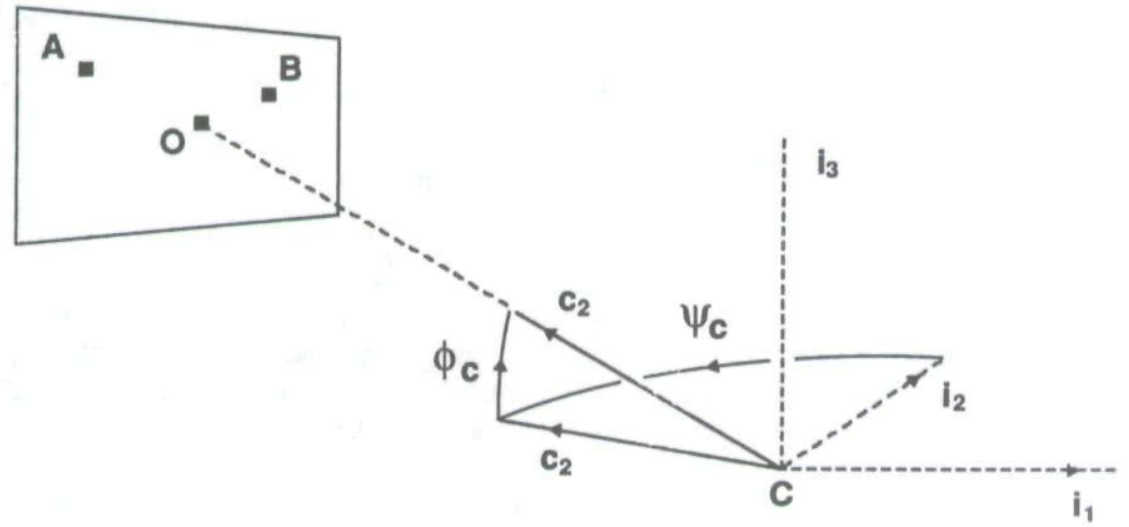

Figure 1 - Pan and tilt angles $\psi_{\mathrm{c}}$ and $\phi_{\mathrm{c}}$ of camera C. 
$\phi_{\mathrm{c}}$. Misalignments of the pan and tilt head and projection head may be taken into account by introducing the screw angle $\theta_{\mathrm{c}}$ about the optical axis $c_{2}$.

The rotation matrix that will transform i-coordinates into c-coordinates is given by,

$$
S_{\text {ic }}=R_{1}\left(\phi_{c}\right) R_{2}\left(\theta_{c}\right) R_{3}\left(\psi_{c}\right)
$$

where the rotation matrices $R_{1}, R_{2}, R_{3}$ are defined by,

$$
\begin{aligned}
& \mathbf{R}_{1}\left(\phi_{\mathrm{c}}\right)=\left[\begin{array}{lll}
1 & 0 & 0 \\
0 & \cos \phi_{\mathrm{c}} & \sin \phi_{\mathrm{c}} \\
0 & \sin \phi_{\mathrm{c}} & \cos \phi_{\mathrm{c}}
\end{array}\right] \\
& \mathbf{R}_{2}\left(\theta_{\mathrm{c}}\right)=\left[\begin{array}{lll}
\cos \theta_{\mathrm{c}} & 0 & \sin \theta_{\mathrm{c}} \\
0 & 1 & 0 \\
\sin \theta_{\mathrm{c}} & 0 & \cos \theta_{\mathrm{c}}
\end{array}\right] \\
& \mathbf{R}_{3}\left(\psi_{\mathrm{c}}\right)=\left[\begin{array}{lll}
\cos \psi_{\mathrm{c}} & \sin \psi_{\mathrm{c}} & 0 \\
-\sin \psi_{\mathrm{c}} & \cos \psi_{\mathrm{c}} & 0 \\
0 & 0 & 1
\end{array}\right]
\end{aligned}
$$

The orientation of the optical axis $\overline{\mathrm{CO}}$ joining camera $\mathrm{C}$ to the film frame center $\mathrm{O}$ is given by the pan angle $\psi_{\mathrm{c}}$ and the tilt angle $\phi_{\mathrm{c}}$ (Figure 1). In the same way, the orientation of the line $\overline{\mathrm{CA}}$ joining camera $\mathrm{C}$ to reference marker $\mathrm{A}$ (Figure 1) may be defined by pan and tilt angles $\psi_{\mathrm{a}}, \phi_{\mathrm{a}}$. These angles specify a frame of reference $\mathrm{A}$, and the rotation matrix $\mathrm{S}_{\mathrm{ai}}$ will transform from $\mathrm{A}$ to frame I where,

$$
\mathrm{S}_{\mathrm{ai}}=\mathrm{R}_{3}\left(-\psi_{\mathrm{a}}\right) \mathrm{R}_{\mathbf{1}}\left(-\phi_{\mathrm{a}}\right)=\left[\begin{array}{ccc}
\cos \psi_{\mathrm{a}} & -\sin \psi_{\mathrm{a}} \cos \phi_{\mathrm{a}} & \sin \psi_{\mathrm{a}} \sin \phi_{\mathrm{a}} \\
\sin \psi_{\mathrm{a}} & \cos \psi_{\mathrm{a}} \cos \phi_{\mathrm{a}} & -\cos \psi_{\mathrm{a}} \sin \phi_{\mathrm{a}} \\
0 & \sin \phi_{\mathrm{a}} & \cos \phi_{\mathrm{a}}
\end{array}\right]
$$

In frame A the column vector [ [ $\left.\begin{array}{lll}0 & 1 & 0\end{array}\right] \mathrm{T}$ corresponds to axis $a_{2}$ which is a unit vector parallel to $\overline{\mathrm{CA}}$. Since $\mathrm{S}_{\mathrm{ai}}\left[a_{2}\right]_{\mathrm{a}}=\left[a_{2}\right]_{\mathrm{i}}$ where []$_{\mathrm{a}}$ denotes the components in frame A,

$$
\mathrm{S}_{\mathrm{ai}}=\left[\begin{array}{l}
0 \\
1 \\
0
\end{array}\right]=\left[\begin{array}{l}
\mathrm{a}_{2}(1) \\
\mathrm{a}_{2}(2) \\
\mathrm{a}_{2}(3)
\end{array}\right]
$$

That is,

$$
\left[\begin{array}{l}
-\sin \psi_{\mathrm{a}} \cos \phi_{\mathrm{a}} \\
\cos \psi_{\mathrm{a}} \cos \phi_{\mathrm{a}} \\
\sin \phi_{\mathrm{a}}
\end{array}\right]=\left[\begin{array}{l}
\mathrm{a}_{2}(1) \\
\mathrm{a}_{2}(2) \\
\mathrm{a}_{2}(3)
\end{array}\right]
$$

If the locations of $\mathrm{C}$ and $\mathrm{A}$ are known, Equation 6 may be used to obtain angles $\phi_{\mathrm{a}}$ and $\psi_{\mathrm{a}}$. The same procedure may be used to calculate angles $\phi_{\mathrm{b}}$ and $\psi_{b}$ for reference marker B. These angles may now be used together with the digitized coordinates of points $\mathrm{A}$ and $\mathrm{B}$ to calculate the orientation angles of camera $\mathrm{C}$ in the following way:

Suppose that a translation of origin is made so that the film frame center $\mathrm{O}$ has digitizer coordinates $(0,0)$, marker $\mathrm{A}$ has coordinates $(\mathrm{M}(1), \mathrm{M}(3))$, and marker B has coordinates $(\mathrm{N}(1), \mathrm{N}(3))$. The camera angles may be initially approximated as, 


$$
\begin{aligned}
\psi_{\mathrm{c}} & =\left[\mathrm{M}(1) \psi_{\mathrm{b}}-\mathrm{N}(1) \psi_{\mathrm{a}}\right] /[\mathrm{M}(1)-\mathrm{N}(1)] \\
\phi_{\mathrm{c}} & =\left[\mathrm{M}(3) \phi_{\mathrm{b}}-\mathrm{N}(3) \phi_{\mathrm{a}}\right] /[\mathrm{M}(3)-\mathrm{N}(3)] \\
\theta_{\mathrm{c}} & =0
\end{aligned}
$$

Equations 7 and 8 are based on the assumption that angular distances are approximately proportional to digitized distances. The approximations given by Equations 7 and 8 will be good when A and B lie in opposite quadrants of the image so that interpolation rather than extrapolation is used. The initial approximations will be poor when the denominators of the expressions are small.

The rotation matrix $\mathrm{S}_{\mathrm{ic}}$ transforms $\mathrm{i}$-coordinates into c-coordinates so that,

$$
\mathrm{S}_{\mathrm{ic}}[\overline{\mathrm{CA}}]_{\mathrm{i}}=[\overline{\mathrm{CA}}]_{\mathrm{c}}
$$

Thus,

$$
S_{i c}\left[\begin{array}{l}
a_{2}(1) \\
a_{2}(2) \\
a_{2}(3)
\end{array}\right]=k_{a}\left[\begin{array}{l}
M(1) \\
F \\
M(3)
\end{array}\right]
$$

where $a_{2}$ is a unit vector parallel to $\overline{\mathrm{CA}}, \mathrm{F}$ is a pseudo focal length in digitizer units, and $\mathrm{k}_{\mathrm{a}}$ is a constant. This equation may be written in the form,

$$
\left[\begin{array}{l}
\mathrm{a}(1) \\
\mathrm{a}(2) \\
\mathrm{a}(3)
\end{array}\right]=\mathrm{k}_{\mathrm{a}}\left[\begin{array}{l}
\mathrm{M}(1) \\
\mathrm{F} \\
\mathrm{M}(3)
\end{array}\right]
$$

where the unit vector $a=\mathrm{S}_{\mathrm{ic}}\left[a_{2}\right]_{\mathrm{i}}=\left[a_{2}\right]_{\mathrm{c}}$.

The same procedure is used for point $\mathrm{B}$, giving,

$$
\left[\begin{array}{l}
\mathrm{b}(1) \\
\mathrm{b}(2) \\
\mathrm{b}(3)
\end{array}\right]=\mathrm{kb}_{\mathrm{b}}\left[\begin{array}{l}
\mathrm{N}(1) \\
\mathrm{F} \\
\mathrm{N}(3)
\end{array}\right]
$$

Equations 12 and 13 provide four estimates of the pseudo focal length $F$. The estimate most likely to be good is chosen as follows:

If $|a(1)-b(1)|$ is smaller than $|a(3)-b(3)|$, the error in $\psi_{c}$ from Equation 7 will be greater than the error in $\phi_{\mathrm{c}}$ from Equation 8. The error in $\psi_{\mathrm{c}}$ will produce systematic errors in $a(1)$ and $b(1)$. In this case $a(3)$ and $b(3)$ are used in the calculation of $F$. If $|a(3)|$ is smaller than $|b(3)|$, the corresponding estimate of $\mathrm{F}$ is less likely to be accurate. $\mathrm{F}$ may then be obtained from Equation 13 as,

$$
\mathrm{F}=\mathrm{b}(2) \mathrm{N}(3) / \mathrm{b}(3)
$$

This procedure avoids the problems that occur when $|\mathrm{a}(1)-\mathrm{b}(1)|$ or $\mathrm{a}(3)$ are close to zero. The remaining three cases are treated in a similar manner.

Since $\mathrm{F}$ should be the same for each film frame, a mean value over all frames is calculated. This value is then used in the recalculation of the camera angles for each film frame. The values given by Equations 7, 8, and 9 for $\psi_{\mathrm{c}}, \phi_{\mathrm{c}}$, and $\theta_{\mathrm{c}}$ are used as initial values in the following iterative procedure:

Equation 11 may be written in the form,

$$
\mathrm{R}_{1}\left(\phi_{\mathrm{c}}\right)\left[\begin{array}{l}
\mathrm{a}(1) \\
\mathrm{a}(2) \\
\mathrm{a}(3)
\end{array}\right]=\mathrm{k}_{\mathrm{a}}\left[\begin{array}{l}
\mathrm{M}(1) \\
\mathrm{M}(2) \\
\mathrm{M}(3)
\end{array}\right]
$$


where $a=\mathrm{R}_{2}\left(\theta_{\mathrm{c}}\right) \mathrm{R}_{3}\left(\psi_{\mathrm{c}}\right)\left[a_{2}\right]_{\mathrm{i}}$ and $\mathrm{M}(2)=\mathrm{F}$.

$\phi_{\mathrm{c}}$ may be obtained from Equation 15 as,

$$
\tan \phi_{\mathrm{c}}=[\mathrm{M}(2) \mathrm{a}(3)-\mathrm{M}(3) \mathrm{a}(2)] /[\mathrm{M}(2) \mathrm{a}(2)+\mathrm{M}(3) \mathrm{a}(3)]
$$

Equation 11 may also be written in the form,

$$
\mathrm{R}_{3}\left(\psi_{\mathrm{c}}\right)\left[a_{2}\right]_{\mathrm{i}}=\mathrm{k}_{\mathrm{a}} \mathrm{R}_{2}\left(-\theta_{\mathrm{c}}\right) \mathrm{R}_{1}\left(-\phi_{\mathrm{c}}\right)[M]_{\mathrm{c}}
$$

and $\psi_{\mathrm{c}}$ may be determined.

The angles $\phi_{\mathrm{c}}$ and $\psi_{\mathrm{c}}$ may also be calculated using the reference marker $B$ and its image (N(1), N(3)). The angles are evaluated iteratively using the average of the values obtained from A and B at each stage. The value of angle $\theta_{c}$ is adjusted at each iteration by comparing the actual direction of the line $\overline{M N}$ with the expected direction calculated from the transformation matrix $S_{i c}$ and the vectors $\overline{\mathrm{CA}}$ and $\overline{\mathrm{CB}}$. The matrix $\mathrm{S}_{\mathrm{ic}}$ which transforms coordinates from the inertial frame I to the camera frame $\mathrm{C}$ is calculated in each iteration using Equation 1. The inverse transformation matrix $\mathrm{S}_{\mathrm{ci}}$ is the transpose of $\mathrm{S}_{\mathrm{ic}}$.

If $\mathrm{P}$ is a body landmark with digitized coordinates $(\mathrm{Q}(1), \mathrm{Q}(3))$, the unit vector $p_{2}$ parallel to line $\overline{\mathrm{CP}}$ joining camera and landmark may be evaluated as,

$$
\left[p_{2}\right]_{\mathrm{i}}=\mathrm{S}_{\mathrm{ci}}\left[\begin{array}{l}
\mathrm{Q}(1) \\
\mathrm{F} \\
\mathrm{Q}(3)
\end{array}\right]
$$

The direction vector $\overline{\mathrm{SP}}$ from a second camera $\mathrm{S}$ to the landmark $\mathrm{P}$ may be found in the same way. An estimate of the location of $P$ is then obtained by taking the midpoint of the common perpendicular to the rays $\overline{\mathrm{CP}}$ and $\overline{\mathrm{SP}}$, providing these rays correspond to the same moment in time.

\section{Synchronization}

In order to obtain digitized data sets that are synchronized, two moments in time are identified for the film from cameras $\mathrm{C}$ and $\mathrm{S}$. If the first film frame digitized is the first frame of the flight phase, the first frames of cameras C and S should differ by less than the interval between frames. Prior to takeoff, the skis of the jumper lie in snow tracks laid down by forerunners. These tracks straddle a vertical plane that bisects the inrun so that at takeoff this plane will lie very close to the sagittal plane of the jumper. Let the rays $\mathrm{CP}_{1}, \mathrm{CP}_{3}$, and $\mathrm{SP}_{2}$ from the cameras to the ski jumper's center of mass meet this vertical plane $V$ in the points $P_{1}$, $P_{3}$, and $P_{2}$ (Figure 2). Points $P_{1}$ and $P_{3}$ correspond to Frames 1 and 3 of camera $C$ while $\mathrm{P}_{2}$ corresponds to Frame 2 of camera $\mathrm{S}$. The ratio in which $R$, the projection of $P_{2}$ on $P_{1} P_{3}$, divides $P_{1} P_{3}$ is then used to determine the time of Frame 2 of camera $\mathrm{S}$ in terms of the time scale of camera $\mathrm{C}$.

This first method cannot be used to synchronize the frames near landing since the jumper may no longer lie in the vertical plane $\mathrm{V}$. Let $c_{\mathrm{n}}$ and $c_{\mathrm{n}-2}$ be rays from camera $\mathrm{C}$ to the ski jumper's center of mass for the last frame and the second frame earlier, and let $s_{\mathrm{n}-1}$ be the ray from camera $\mathrm{S}$ to the center of mass using the penultimate frame of camera $S$ (Figure 3). Let the ray $s_{n-1}$ meet the plane of $c_{\mathrm{n}}$ and $c_{\mathrm{n}-2}$ at the point $\mathrm{P}_{\mathrm{n}-1}$. The ratio in which $\mathrm{CP}_{\mathrm{n}-1}$ divides the angle formed by $c_{\mathrm{n}}$ and $c_{\mathrm{n}-2}$ is then used to determine the time of the penultimate frame of camera $\mathrm{S}$ in terms of the time scale of camera $\mathrm{C}$. It should be noted that this second method of synchronization will be accurate if the angle 


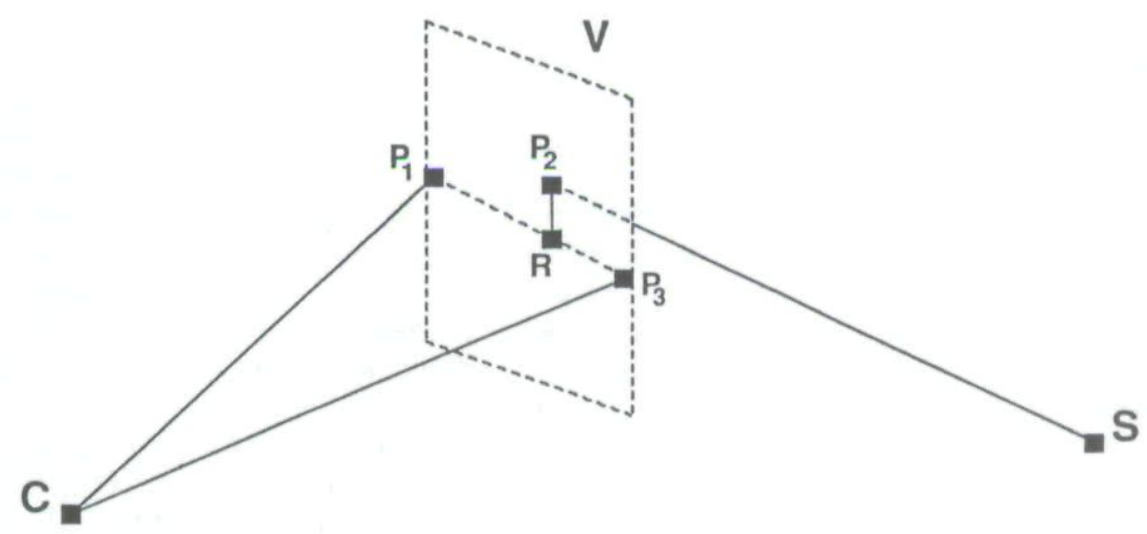

Figure 2 - Synchronization of cameras $\mathrm{C}$ and $\mathrm{S}$ at takeoff.

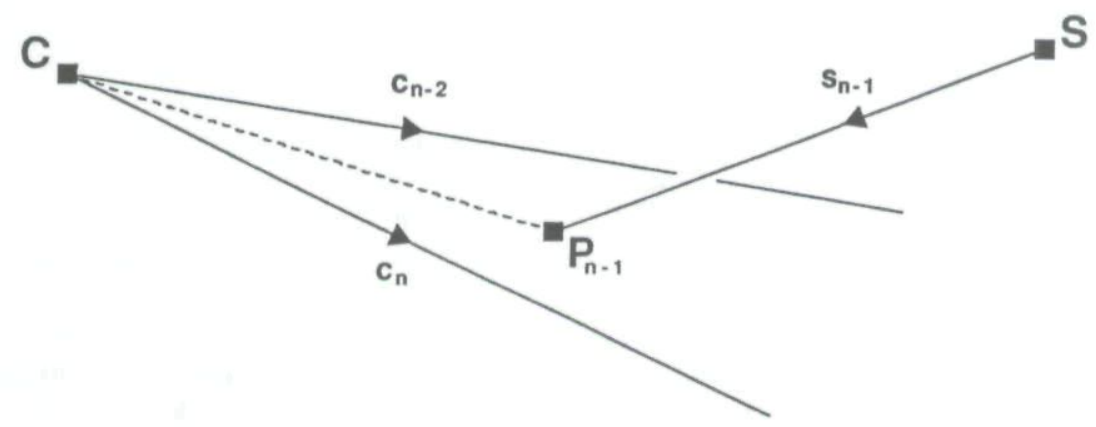

Figure 3 - Synchronization of cameras $\mathrm{C}$ and $\mathrm{S}$ at landing.

between the ray $s_{\mathrm{n}-1}$ and the plane between $c_{\mathrm{n}}$ and $c_{\mathrm{n}-2}$ approaches a right angle. This will not be the case for the ski jump takeoff if the jumper's velocity vector and the two camera positions are approximately coplanar. Having determined the times for Frames 2 and $n-1$ of camera $S$, the times of the remaining frames are calculated assuming that the frame rate is constant.

\section{Error Estimates}

The position and orientation of the ski jumper may be specified by the location of the mass center together with orientation angles describing orientation and configuration of the body. In order to obtain error estimates of these quantities, the films from cameras $\mathrm{C}$ and $\mathrm{S}$ are digitized twice so that four estimates of each coordinate and each angle are obtained.

The repeated digitization permits error estimates to be obtained for the digitized coordinates of the body landmarks so that quintic splines may be fitted to the digitized data (Wood \& Jennings, 1979). The repeated digitization using differ- 
ent reference markers allows the calculation of two independent sets of camera angles. These angles are fitted with quintic splines using error estimates based on the differences between the two calculations. The quintic splines are evaluated at 101 equally spaced times to provide synchronized sets of digitized data and camera angles.

The three-dimensional coordinates of each body landmark are then evaluated using the four combinations of synchronized data. These four sets of threedimensional coordinates are used to calculate four (dependent) sets of estimates of the coordinates of the center of mass and the orientation angles of the body (Yeadon, in press-a). If $\mathrm{A}_{\mathrm{ij}}$ is the estimate of orientation angle $\mathrm{A}$ using the ith digitization of camera $C(i=1,2)$ and the jth digitization of camera $S(j=1,2)$, then $A_{11}$ and $A_{22}$ will be based upon independent digitizations and similarly for $A_{12}$ and $A_{21}$. On the other hand, $A_{11}$ and $A_{12}$ both use the first digitization of camera $\mathrm{C}$ and therefore are not independent. An unbiased estimate of the variance $v$ of the mean $A$ of four estimates $A_{11}, A_{12}, A_{21}$, and $A_{22}$ is calculated using,

$$
v=\left[\left(A_{11}-A_{22}\right)^{2}+\left(A_{12}-A_{21}\right)^{2}\right] / 8
$$

as described in Yeadon (in press-a). The overall variance is obtained by averaging over the 101 different times, and a global standard error estimate of each mean is calculated.

\section{Data Collection and Analysis}

Prior to competition, the small hill (70-m jump) at Canada Olympic Park in Calgary was surveyed using a combination of conventional and photogrammetric survey methods. Reference markers comprised $10-\mathrm{cm}$ diameter styrofoam spheres. The conventional survey determined the locations of the three photogrammetric camera stations, the two camera platforms to be used by the cine cameras, and nine spherical reference markers. Images of the landing hill were obtained on glass plates using a Zeiss-Jena UMK-1 metric camera from the three photogrammetric stations. Photogrammetric measurements of 179 landmarks were made monoscopically using a Wild $\mathrm{ACl}$ analytical stereoplotter. These measurements, together with the locations of the photogrammetric stations and four of the nine conventionally surveyed markers and the metric camera calibration, were used to calculate the locations of the landmarks. The remaining five conventionally surveyed markers were used as an independent check on the accuracy of the calculated coordinates.

Four camera operators practiced following the ski jumpers with cameras mounted on tripods with pan and tilt heads during training sessions. Two of these four operators proved able to track the flight reliably, one operator using throughthe-lens viewing and the other lining up one edge of the camera with the jumper. The 70-meter Olympic ski jumping competition was filmed using two Locam cameras equipped with 100-200-mm zoom lenses (Figure 4). Focal lengths were set at about $130 \mathrm{~mm}$, and camera-to-jumper distances ranged from $100 \mathrm{~m}$ to $180 \mathrm{~m}$ so that the width of field ranged from about $8 \mathrm{~m}$ to $14 \mathrm{~m}$. Camera frame rates were set to nominal rates of $45 \mathrm{fps}$ and variable shutters were set to $1 / 6$. The pan and tilt heads on the tripods were leveled using a built-in spirit level. The camera operators were linked by telephone to the director, who gave the start and stop orders for filming each jumper. Locations of camera lenses relative to the camera platforms were determined using plumb line and steel tape measure. 


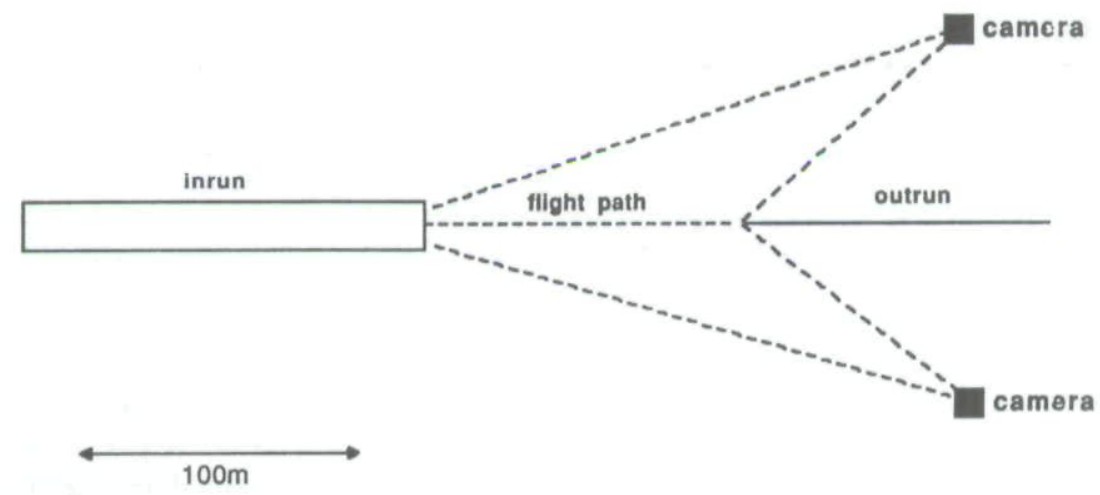

Figure 4 - Camera locations.

Films of three jumpers were digitized using an SAC GP-8 Sonic Digitizer measuring $1.0 \mathrm{~m}$ by $0.6 \mathrm{~m}$. The number of frames per jump ranged from 98 to 157 frames, and each view took up to 3 hours to digitize. In each frame, 12 body landmarks were digitized as well as two reference markers. The body landmarks used were wrist, shoulder, hip and ankle centers, and front and rear ski tips. This permitted a seven-segment representation of the jumper as trunk, two arms, two legs, and two skis.

Anthropometric data were collected on five participants in the Nordic Combined competition. For each of the three ski jumpers filmed, the participants closest in height and weight were chosen and segmental masses and mass centers were obtained using an inertia model (Yeadon, in press-b). A pair of skis and boots were weighed, balanced, and measured to determine masses and mass centers.

The orientation of the trunk in space was defined by successive rotations about lateral, anterior/posterior, and longitudinal axes corresponding to somersault, tilt, and twist (Yeadon, in press-a). The configuration of body segments was defined using two angles to specify the orientation of each nontrunk segment relative to the adjacent proximal segment. The time histories of the three coordinates of each jumper's mass center, 3 angles describing orientation, and 12 angles describing body configuration were calculated from the digitized data, the surveyed points, and the segmental masses and mass centers.

\section{Results and Discussion}

The global error estimate of the coordinates of the surveyed reference markers was calculated to be $0.03 \mathrm{~m}(1 S D)$. An estimate of the error in synchronizing the cameras was obtained by comparing the ratio of the framing rates determined by the internal timing lights with the ratio given by the synchronization calculation. This error estimate was $0.005 \mathrm{sec}$. Error estimates of the digitized data, pseudo focal lengths, and camera angles are given in Table 1. Digitized displacement values were obtained in meters by scaling so that the width of the film frame was $10 \mathrm{~m}$, which is close to the average field of view in the plane containing the ski jumper. The values obtained indicate that repeated estimations of joint 
centers lie within about $1 \mathrm{~cm}$ of each other. It is possible that this value could be reduced slightly if a longer focal length lens were used, but much of the error probably arises from the difficulty in visually estimating joint centers regardless of the image size. The error in the pseudo focal length estimate is around $0.5 \%$ and arises from errors in digitizing the markers in the survey of the markers. The errors in the pan and tilt angles are close to $0.02^{\circ}$, and for a camera-marker distance of $150 \mathrm{~m}$ this corresponds to an error of $0.05 \mathrm{~m}$ in locating the marker. This value could be reduced by narrowing the shutter angle to reduce the marker blur arising from panning and ensuring that all markers are rigidly attached to immovable objects.

Error estimates of the xyz-components of the mass center and of the orientation angles for each jumper are given in Table 2. The largest errors in the location of the mass center occur in the y-coordinate which describes the horizontal forward displacement. This is to be expected since the narrow angle between the camera axes at takeoff (Figure 4) will result in larger errors in the y-direction. Since these error estimates were determined using repeated digitization, they should be regarded as underestimates because they do not account for systematic errors. Errors in determination of the camera locations, the nonlinearity of camera

Table 1

\section{Error Estimates of Data}

Trial number

$\begin{array}{lll}24 & 37 & 47\end{array}$

\begin{tabular}{llll} 
Digitized coordinates & $0.009 \mathrm{~m}$ & $0.012 \mathrm{~m}$ & $0.010 \mathrm{~m}$ \\
Pseudo focal length & $0.35 \%$ & $0.36 \%$ & $0.60 \%$ \\
Pan angle & $0.02^{\circ}$ & $0.02^{\circ}$ & $0.02^{\circ}$ \\
Screw angle & $0.05^{\circ}$ & $0.06^{\circ}$ & $0.14^{\circ}$ \\
Tilt angle & $0.02^{\circ}$ & $0.02^{\circ}$ & $0.02^{\circ}$ \\
\hline
\end{tabular}

Table 2

Error Estimates of Coordinates and Angles

\begin{tabular}{llll}
\hline & \multicolumn{3}{c}{ Trial number } \\
\cline { 2 - 4 } & \multicolumn{1}{c}{34} & 47 \\
\hline x coordinate & $0.011 \mathrm{~m}$ & $0.015 \mathrm{~m}$ & $0.023 \mathrm{~m}$ \\
y coordinate & $0.021 \mathrm{~m}$ & $0.031 \mathrm{~m}$ & $0.045 \mathrm{~m}$ \\
z coordinate & $0.013 \mathrm{~m}$ & $0.012 \mathrm{~m}$ & $0.016 \mathrm{~m}$ \\
Angles & $0.9^{\circ}$ & $0.7^{\circ}$ & $0.9^{\circ}$ \\
\hline
\end{tabular}

Note. $\mathrm{x}$-axis is right-left; $\mathrm{y}$-axis is forward; $\mathrm{z}$-axis is downward. 

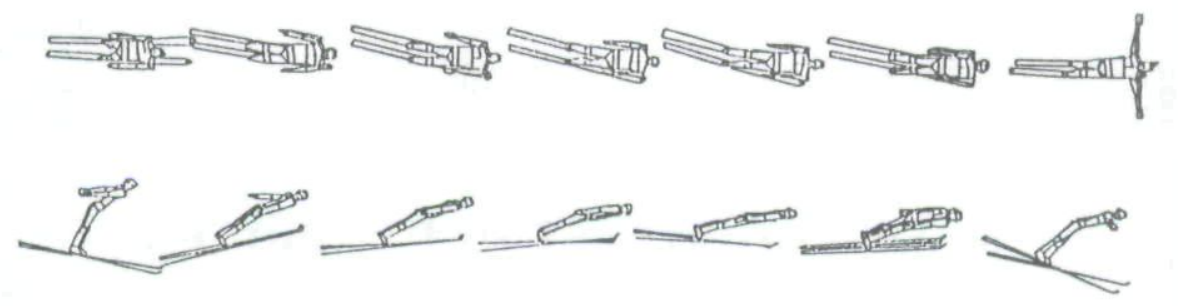

Figure 5 - Orientation during flight.

and projection lenses, and distortions of the film will lead to additional errors in the center of mass estimates. Such systematic errors are likely to have a small effect on the calculated orientation angle values since each joint center will incur a similar systematic error at a given time. The error estimates of the 15 orientation angles average less than $1^{\circ}$.

Figure 5 shows side and top views of the body orientations in the final jump of the winning competitor. The turning of the skis out of the sagittal plane during flight can be seen. Half a second before landing, the jumper and skis have twisted to the left but this appears to have been corrected before landing. The threedimensional nature of ski jumping is apparent and the presented method provides a means of investigating ski jumping technique.

\section{References}

Abdel-Aziz, Y.I., \& Karara, H.M. (1971). Direct linear transformation from comparator coordinates into object space coordinates in close-range photogrammetry. ASP Symposium on Close Range Photogrammetry. Falls Church, VA: American Society of Photogrammetry.

Bergemann, B.W. (1974). Three-dimensional cinematography: A flexible approach. Research Quarterly, 45, 302-309.

Dapena, J. (1978). Three-dimensional cinematography with horizontally panning cameras. Sciences et Motricite, 1(3), 3-15.

Penrose, T., Wood, G., \& Blanksby, B. (1976). The accuracy of positional data in triaxial cinematography. Australian Journal for Health, Physical Education and Recreation, 71, 7-12.

Van Gheluwe, B. (1978). Computerized three-dimensional cinematography for any arbitrary camera setup. In E. Asmussen \& K. Jorgensen (Eds.), Biomechanics VI (pp. 343-348). Baltimore: University Park Press.

Wood, G.A., \& Jennings, L.S. (1979). On the use of spline functions for data smoothing. Journal of Biomechanics, 12, 477-479.

Yeadon, M.R. (in press-a). The simulation of aerial movement. Part I: The determination of orientation angles from film data. Journal of Biomechanics.

Yeadon, M.R. (in press-b). The simulation of aerial movement. Part II: A mathematical inertia model of the human body. Journal of Biomechanics.

We gratefully acknowledge the support of the IOC Medical Commission, the Natural Sciences and Engineering Research Council, the International Ski Federation, CTV Television, Red Lake Corp., XV Olympic Winter Games Organizing Committee, and The University of Calgary. 